\title{
Endogenous Information Acquisition for an Overconfident Retailer
}

\author{
Jialu Li, Meiying Yang \\ School of Economics and Management, Beihang University, Beijing, China \\ Email: lj120@foxmail.com, myang@buaa.edu.cn
}

How to cite this paper: Li, J.L. and Yang, M.Y. (2018) Endogenous Information Acquisition for an Overconfident Retailer. Modern Economy, 9, 422-433. https://doi.org/10.4236/me.2018.93027

Received: January 30, 2018

Accepted: March 12, 2018

Published: March 15, 2018

Copyright $\odot 2018$ by authors and Scientific Research Publishing Inc. This work is licensed under the Creative Commons Attribution International License (CC BY 4.0).

http://creativecommons.org/licenses/by/4.0/

\begin{abstract}
Overconfidence has been proven to be "one of the most consistent, powerful and wide spread cognitive biases". In this paper, we develop a model in which a supplier selling to an overconfident retailer invests in acquiring market information. Overconfidence causes the retailer to overestimate the precision of his private information as well as his capacity to acquire information. Our work shows that overconfidence hurts the retailer's profits, and may even eliminate the benefits of information. Instead, the supplier can benefit from the retailer's overconfidence. We demonstrate that this bias with an endogenous information-acquisition effort can coordinate the supply chain to achieve its first-best benchmark.
\end{abstract}

\section{Keywords}

Overconfidence, Information Acquisition, Supply Chain Coordination

\section{Introduction}

Escalating levels of demand uncertainty, caused by globalization, outsourcing, shorter product lifecycles, and the proliferation of goods and categories, are a general phenomenon in the contemporary business environment. Information obtained from market research can reduce the uncertainty, and then becomes a key driver for improving supply chain performance. However, information acquisition can be costly in terms of time, money, and human resources, which have frequently been recognized by both practitioners [1] and academics [2]. With the explosion in both the scope and volume of customer and market data, for example, companies need to use professional software programs, train in-house market research personnel, or hire outside consulting experts to analyze such data and obtain reliable market information. Therefore, an important 
decision making problem in those situations is how much information should firms collect? A large research based on the models with rational firms gives the answers to this question. The investors (firms) should spend money and time acquiring information up to the point where the marginal benefit of doing so exceeds the marginal cost [3]-[8].

However, findings gathered from diverse disciplines, such as economic and finance and management have identified that individual cognitive bias can influence the decision-making process in complex and uncertain environments [9] [10]. Among various known psychological biases, overconfidence has been proven to be one of the most consistent, powerful, and widespread biases [11]. Apart from the field of psychology [12], this bias has also been observed in a wide range of fields from economics [13] to finance [14] to operations management [15]. In addition, researchers have identified that the bias of overconfidence is relevant to the pull-to-center and bullwhip problems in a managerial context [16] [17].

With respect to information acquisition, a large body of evidence from experimental research shows that many investors are overconfident when they make acquisition decisions [18]. Specifically, overconfident forecasters overestimate not only the value (precision) of the private signals [19] [20], but also the productivity in their investment in information [21]. They invest resources in acquiring information in spite of it being unclear that they can even achieve returns that recoup these costs. As a result, they may attain poorer performance.

Although information acquisition has been studied extensively in operations research/operations management, the existing model in literature has largely ignored the cognitive bias (overconfidence in particular) of firms. Therefore, our research explores the consequences of overconfidence for information acquisition investment, aiming to establish whether this bias would eliminate the benefits of information. To achieve this, we develop a behavior game model that incorporates the notion of overconfidence into the information acquisition model pioneered by $\mathrm{Fu}$ and $\mathrm{Zhu}$ [6], which has a single supplier selling to an overconfident retailer that can acquire costly demand information. With overconfidence, the retailer overestimates the precision of his private information as well as his capacity to acquire information. Below we summarize our main results and insights.

First, we study how overconfidence causes the information acquisition level to deviate from the first-best benchmark. We find overconfidence can make the retailer overinvest in information acquisition. Second, our analysis shows overconfidence is harmful to the retailer, and excessive overconfidence may even eliminate the benefits of information. But the supplier can benefit from selling to a more biased retailer. Finally, we demonstrate overconfidence with endogens information acquisition can coordinate the supply chain if the total profit margin of a product is low.

The rest of the paper is organized as follows. We introduce the model in Sec- 
tion 2. Section 3 derives the optimal decision in the integrated setting. Section 4 provides the equilibrium analysis for the overconfident retailer in the decentralized setting, and compares integrated case and decentralized case. Based on the analysis above, Section 5 analyzes whether channel coordination can be achieved by overconfidence. Section 6 concludes the paper.

\section{Model}

We consider a supply chain consisting of a supplier (she) and an over confident retailer (he). The supplier produces a product and sells it to the retailer, who faces a random demand, $D \sim N\left(\mu_{D}, \sigma_{D}^{2}\right)$, during a single selling season. The retailer has an opportunity to acquire a private signal $x$ about the market condition. Assume that $x=D+\varepsilon$, where $\varepsilon$ is independent of $D$ with $\varepsilon \sim N\left(0, \sigma_{\varepsilon}^{2}\right)$. Let $\sigma^{2} \equiv \sigma_{\varepsilon}^{2} /\left(\sigma_{\varepsilon}^{2}+\sigma_{D}^{2}\right)$. From the conjugate property of the normal distribution, the posterior distribution of $D$ for a given signal $x$ is

$$
D \mid x \sim N\left(\mu_{x}, \sigma_{x}^{2}\right)
$$

where $\mu_{x}=\sigma^{2} \mu_{D}+\left(1-\sigma^{2}\right) x$ and $\sigma_{x}^{2}=\sigma_{D}^{2} \sigma^{2}$.

Note that $\sigma$ is a measure of the signal's precision in predicting in market condition. The larger the value of $\sigma$, the less information the signal has about the market condition. If $\sigma=1$, the posterior distribution of the market condition is identical to its prior distribution; i.e., the signal contains no useful information. On the other hand, as $\sigma \rightarrow 0$, the signal reveals the exact value of the market condition.

The precision of acquired information depends on the retailer's investment in information acquisition. We assume the retailer incurs a cost of $v$ for obtaining a signal that has a precision

$$
\sigma(v) \equiv \frac{k}{k+v}
$$

From (2), the precision function is decreasing and convex in $v$ and that zero investment generates no information, i.e.,

$$
\frac{\partial \sigma}{\partial v}<0, \frac{\partial^{2} \sigma}{\partial v^{2}}>0 \text { and } \sigma(0)=1 \text {. }
$$

The parameter $k$ measures the capability of acquiring information. Given the investment $v$, the retailer with a smaller value of $k$ can acquire the more accurate signal.

Because of overconfidence, however, the retailer not only overestimates the precision of her information but also the capability to acquire information [21]. We assume that the retailer's subjective precision has the following functional form:

$$
\beta(\alpha, v)=\frac{k(1-\alpha)}{k(1-\alpha)+v},
$$

where her level of overconfidence is given by the parameter $\alpha \in[0,1]$. The case 
$\alpha=0$ represents the benchmark of rationality, and the overconfident retailer has $\eta>0$. Given the investment $v$, the retailer believes the precision of $x$ to be $\beta(\alpha, v)<\beta(0, v)=\sigma(v)$ and the updated demand distribution is $D \mid x \sim N\left(\beta^{2} \mu_{D}+\left(1-\beta^{2}\right) x, \sigma_{D}^{2} \beta^{2}\right)$.

Because there is a tradeoff between the cost of acquiring this information and the benefit obtained from using this information, the overconfident retailer first needs to decide investment level $v$. After acquiring forecasts, the retailer updates her knowledge of the demand, determines the order quantity, $q$, to be placed with the supplier, and makes a payment according to the wholesale price contract $w>c$. The supplier then produces an exact amount of $q$ units, which costs his $c \cdot q$, and delivers them to the retailer. Finally, demand occurs and is filled from the stocked inventory as much as possible, during which the unit selling price is $p>w$. Without loss of generality, we assume a zero penalty cost and zero salvage value, so the overage cost is the cost of procurement, and the underage cost is the loss of profit.

\section{Unbiased Integrated System}

To better understand the effect of overconfidence on information acquisition investment, we first consider a benchmark case in which the supplier and the retailer are owned by an unbiased central decision maker. The central decision maker first decides on the information-acquisition level, then determines the optimal production quantity given the forecast $x$. We solve the problem backwards.

Given $v$ and $x$, the central decision maker determines $q$ to maximize $p E_{D \mid x} \min (D, q)-c q$. Let $\phi(\cdot)$ and $\Phi(\cdot)$ be the probability density function (pdf) and the cumulative density function (cdf) of the standard normal distribution, respectively. From Porteus [22], we obtain the optimal value of $q$ as $q^{I}=\mu_{x}+z^{I} \sigma_{D} \sigma(v)$, where $z^{I}=\Phi^{-1}\left(\frac{p-c}{p}\right)$. Furthermore, we can show

$$
p E_{D \mid x} \min \left(D, q^{I}\right)-c q^{I}=(p-c)\left(\sigma^{2} \mu_{D}+\left(1-\sigma^{2}\right) x\right)-p \sigma_{D} \phi\left(z^{I}\right) \sigma(v) .
$$

By $E[x]=\mu_{D}$, the central decision maker chooses investment level $v$ to maximizes the expected profit

$$
\pi^{I}(v)=(p-c) \mu_{D}-p \sigma_{D} \phi\left(z^{I}\right) \sigma(v)-v .
$$

The second and third term of Equation (4) represents the demand-uncertainty cost and the cost of acquiring information, respectively. The demand-uncertainty cost decreases due to the improvement in information's precision. Hence, the optimal investment level is determined by the tradeoff between the second term and third one, which is

$$
v^{I}=\left\{\begin{array}{cc}
\sqrt{k \Lambda\left(z^{I}\right)}-k, & \text { if } k<\Lambda\left(z^{I}\right) \\
0, & \text { Otherwise }
\end{array}\right.
$$


where $\Lambda(\cdot)=p \sigma_{D} \phi(\cdot)$.

As is clear from (5), the central planner invests in acquiring information only when $k<\Lambda\left(z^{I}\right)$. In other words, if the capability to acquire information is too poor, it is optimal not to acquire any information at all. Further, $\Lambda\left(z^{I}\right)$ is increasing in $p$ and increasing in $c$ for $c \in(0, p / 2)$ and decreasing in $c$ for $c \in(p / 2, p) \quad$ (Fu and Zhu 2010). When the price $p$ is high, the cost $c$ is moderate (close to $p / 2$ ), and the underlying demand is volatile ( $\sigma_{D}$ is large), therefore, larger investments are required in the acquisition of demand information.

\section{Decentralized System}

In this section, we analyze a decentralized setting where the firms make decisions to maximize their own profits. Given the investment level $v$, the overconfident retailer believe he would obtain the signal $x$ with the precision $\beta(v)$. Similar to the analysis above, the biased order quantity is $q^{*}=\mu_{x}+z^{*} \beta \sigma_{D}$, where $z^{*}=\Phi^{-1}\left(\frac{p-w}{p}\right)$. Given investment level $v$, the expected profit of the overconfident retailer is

$$
\pi^{*}(v)=(p-w) \mu_{D}-p \sigma_{D} \phi\left(z^{*}\right) \beta(v)-v .
$$

Proposition 1 indicates how much the overconfident retailer invests in information acquisition.

Proposition 1. (a) The equilibrium investment level for the over confident retailer is

$$
v^{*}(\alpha)=\left\{\begin{array}{cc}
\sqrt{(1-\alpha) k \Lambda\left(z^{*}\right)}-(1-\alpha) k, & \text { if } k<\frac{\Lambda\left(z^{*}\right)}{1-\alpha} \\
0, & \text { otherwise }
\end{array}\right.
$$

(b) If $k>\frac{\Lambda\left(z^{*}\right)}{4}, v^{*}(\alpha)$ is first increasing for

$\alpha \in\left[\max \left\{1-\frac{\Lambda\left(z^{*}\right)}{k}, 0\right\}, 1-\frac{\Lambda\left(z^{*}\right)}{4 k}\right]$ and then decreasing for

$\alpha \in\left[1-\frac{\Lambda\left(z^{*}\right)}{4 k}, 1\right]$. Otherwise, $v^{*}(\alpha)$ decreases with $\alpha \in[0,1]$.

Proof: For (a), consider the profit function stated in (6). It can be verified that $\frac{\mathrm{d}^{2} \pi^{*}(v)}{\mathrm{d} v^{2}}<0$ and $\left.\frac{\mathrm{d} \pi^{*}(v)}{\mathrm{d} v}\right|_{v=0}>0$. Therefore, the optimal investment level $v^{*}$ is the solution to the first order condition, that is $\frac{\mathrm{d} \pi^{*}(v)}{\mathrm{d} v}=0$. For (b), the results directly follow from the (7).

Proposition 1 summarizes the retailer's investment bias in information acqui- 
sition. Similar to the first-best benchmark, if the capacity to acquire information turns out to be too poor, the biased retailer may end up gaining information from market research. Furthermore, the threshold value $\frac{\Lambda\left(z^{*}\right)}{1-\alpha}$ increases with the overconfidence level $\alpha$. It suggests that the retailer is increasingly convinced that he should invest in acquiring information as he becomes more biased.

In addition, the biased investment level is either decreasing or increasing in $\alpha$ depending on the acquisition capacity $k$. If the retailer is already a good forecaster, i.e., $k \leq \Lambda\left(z^{*}\right) / 4$, improvement in overconfidence level makes him feel that he can obtain accurate enough information through less investment due to the (subjectively) stronger acquisition capacity. However, if the retailer has poor forecasting capabilities, i.e., $k>\Lambda\left(z^{*}\right) / 4$, the equilibrium investment level is unimodal in the retailer's overconfidence level. Specifically, the retailer with slight overconfidence ( $\left.\alpha<1-\frac{\Lambda\left(z^{*}\right)}{4 k}\right)$ still believes that he is not a good enough forecaster. In this situation, improvement in overconfidence level gives the retailer the propensity to invest more in information acquisition. Instead, the excessively over confident retailer $\left(\alpha>1-\frac{\Lambda\left(z^{*}\right)}{4 k}\right)$ believes that he is a good forecaster, and then cuts the investment in information acquisition due to the (subjectively) stronger capacity.

To explore the effect of overconfidence on firms' incentives for acquiring information, we focus on the case where $k<\min \left\{\Lambda\left(z^{I}\right), \Lambda\left(z^{*}\right)\right\}$ in the following part.

Lemma 1. For $k<\min \left\{\Lambda\left(z^{I}\right), \Lambda\left(z^{*}\right)\right\}$, we have: (a) If $w>p-c$, $v^{*}(\alpha=0)<v^{I}$; (b) If $w \leq p-c, v^{o}(\alpha=0) \geq v^{I}$. The equation holds when $w=p-c$.

Proof: From (5) and (7), we need to compare $\phi\left(z^{*}\right)$ and $\phi\left(z^{I}\right)$. From Fu and $\mathrm{Zhu}$ (2010), the required results can be obtained.

Lemma 1 shows that for the perfectly unbiased retailer $(\alpha=0)$, the wholesale price plays a key role in determining how the investment level deviates from the first best. Ina case of the wholesale price is relatively low, the retailer can earn a larger profit. Therefore, the unbiased retailer would exert much effort on acquiring demand information than that by a central decision maker. Similar rationale explains why an unbiased retailer invests less than a central planner when the wholesale price is low.

Proposition 2. For $k<\min \left\{\Lambda\left(z^{I}\right), \Lambda\left(z^{*}\right)\right\}$, we have:

(a) When $w<p-c$, there exists $\hat{\alpha}$ such that $v^{*}(\hat{\alpha})=v^{I}$. Moreover, $v^{*}>v^{I}$ for $\alpha \in(0, \hat{\alpha})$ and $v^{*}<v^{I}$ for $\alpha \in(\hat{\alpha}, 1)$.

(b) When $w>p-c, v^{*}(\alpha)>v^{I}$ for some $\alpha$ if $k>\frac{\Lambda\left(z^{*}\right)}{4} \geq v^{I}$. Other- 
wise, $v^{*}<v^{I}$ for any $\alpha \in(0,1)$.

Proof: The results directly follow from Lemma 1 and Proposition 1.

Recall from Proposition 1 that the biased investment level is unimodal or decreasing in overconfidence level. When $w<p-c$, we have $v^{*}(\alpha=0)>v^{I}$ as identified in Lemma 1. In this situation, therefore, the slightly overconfident retailer under-invests in acquiring information, while the excessively overconfident retailer over-invests. On the other hand, under situations so that $v^{*}(\alpha=0)<v^{I}$ as identified in Lemma 1, coupled with an additional condition of $k>\frac{\Lambda\left(z^{*}\right)}{4} \geq v^{I}$, an increasing level of overconfidence pushes $v^{*}$ closer to $v^{I}$, which results in the ultimate crossing of the value of first-best level. Otherwise, the retailer with any level of overconfidence would under-invest in information acquisition.

We next investigate the impact of overconfidence on the performance of the members in the supply chain. Note that $v^{*}(\alpha)=0$ for any $\alpha$ when $k \geq \frac{\Lambda\left(z^{*}\right)}{1-\alpha}$, which is straightforward from Proposition 1. This means that the profit levels of supplier and retailer are independent of retailer's overconfidence level when $k \geq \frac{\Lambda\left(z^{*}\right)}{1-\alpha}$. Therefore, we only consider the case of $k<\frac{\Lambda\left(z^{*}\right)}{1-\alpha}$ in the following proposition.

We first numerically show how overconfidence affects the retailers' performance through undertaking some numerical experiments. We use the following numerical values: $p=4, w \in\{1.5,2,2.5,3\}, \sigma_{D}=3$ and $k=2$. Figure 1(a), Figure 1(c), Figure 1(d) shows that an increasing level of overconfidence hurt the retailer's profit. In Figure 1(b), the retailer's profit increases with $\alpha$ for $\alpha \in[0.4,0.7]$. However, the biased retailer's profit is still smaller than the unbiased one. In general, therefore, overconfidence hurts the retailer's profit. In addition, excessive overconfidence may even eliminate the benefits of information. When $w=3$, for example, the expected profit of the unbiased retailer without information acquisition can be expressed as $\pi^{N}=(p-w) \mu_{D}-p \sigma_{D} \phi\left(z^{*}\right)$ $(\approx 6.18668)$. It can be verified that the expected profit of retailer with information is smaller than the profit without information when $\alpha>0.87$. Therefore, excessive overconfidence could eliminate the benefits of information.

On the other hand, the supplier produces the exact quantity ordered by the retailer and receives an expected profit of

$$
\pi_{S}^{*}=E_{x}\left[(w-c) q^{*}\right]=(w-c)\left(\mu_{D}+z^{*} \sigma_{D} \sqrt{\frac{k(1-\alpha)}{\Lambda\left(z^{*}\right)}}\right) .
$$

Proposition 3. The supplier benefits from the retailer's overconfidence if and only if $w>\frac{p}{2}$.

This proposition shows that overconfidence can be a positive effect on the 

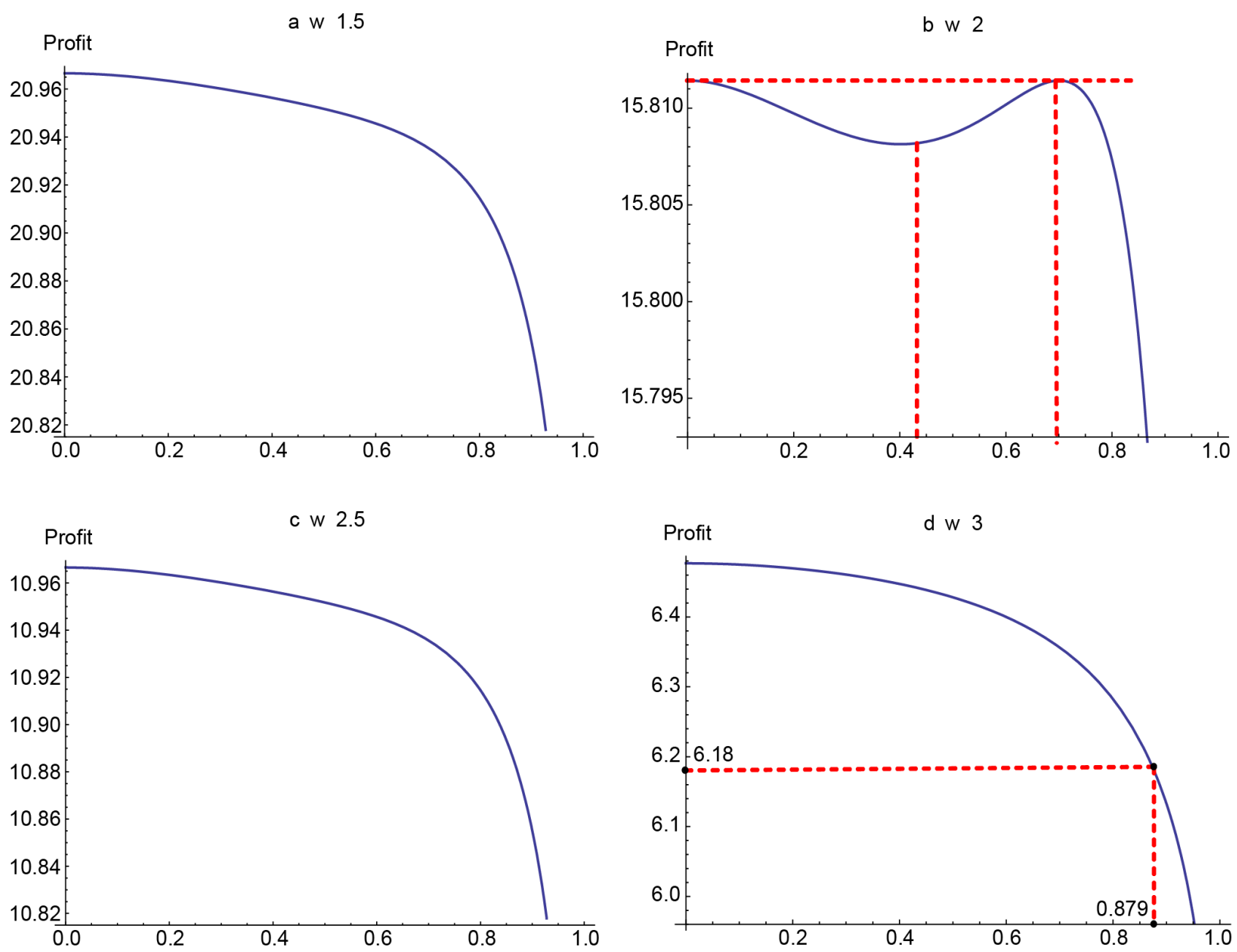

Figure 1. The effect of overconfidence on the retailer's profit.

supplier's performance. When the wholesale price is relatively large, i.e., $w>\frac{p}{2}$, an increasing level of overconfidence boots the supplier's profits.

\section{Channel Coordination}

Under rational player assumption, it is well known that a wholesale price contract cannot coordinate a supply chain due to double marginalization. In this section, we examine whether channel coordination is feasible in the presence of overconfidence. For the first-best benchmark, the equilibrium expected order quantities are

$$
E_{x}\left[q^{I}\right]=\left\{\begin{array}{ll}
\mu_{D}+z^{I} \sigma_{D} \sqrt{\frac{k}{\Lambda\left(z^{I}\right)}}, & \text { if } k<\Lambda\left(z^{I}\right) \\
\mu_{D}+z^{I} \sigma_{D}, & \text { Otherwise }
\end{array} .\right.
$$

In the decentralized system, the equilibrium expected order quantities under the wholesale price contract can be expressed as 


$$
E_{x}\left[q^{*}\right]=\left\{\begin{array}{cc}
\mu_{D}+z^{*} \sigma_{D} \sqrt{\frac{k(1-\alpha)}{\Lambda\left(z^{*}\right)},} & \text { if } k<\frac{\Lambda\left(z^{*}\right)}{1-\alpha} . \\
\mu_{D}+z^{*} \sigma_{D}, & \text { Otherwise }
\end{array}\right.
$$

Proposition 4. When $c \geq \frac{p}{2}$, there exists a level of overconfidence $\alpha^{* *} \in(0,1)$ at which the supply chain can be coordinated under wholesale price contract.

Proof: From (9) and (10), channel coordination can be achieved under wholesale price contract if and only if $E_{x}\left[q^{I}\right]=E_{x}\left[q^{*}\right]$ and $v^{I}=v^{*}(\alpha)$. Therefore, we just need to consider the case of $z^{*}<z^{I}<0$ and $0<z^{*}<z^{I}$.

When $c>\frac{p}{2}$, we have $z^{*}<z^{I}<0$ and $\Lambda\left(z^{*}\right)<\Lambda\left(z^{I}\right)$. In this situation, there are three cases to consider. That is

Case (a): $\Lambda\left(z^{I}\right) \leq k$. There exists $\alpha^{* *} \in\left(1-\frac{\Lambda\left(z^{*}\right)}{k}, 1\right)$ such that

$$
\left(\frac{z^{I}}{z^{*}}\right)^{2}=\frac{k}{\Lambda\left(z^{*}\right)}\left(1-\alpha^{* *}\right)
$$

because $\left(\frac{z^{I}}{z^{*}}\right)^{2}<1$. Hence, $E_{x}\left[q^{I}\right]=E_{x}\left[q^{*}\right]$ when $\alpha=\alpha^{* *}$.

Case (b): $\Lambda\left(z^{*}\right) \leq k<\Lambda\left(z^{I}\right)$. There exists $\alpha^{* *} \in\left(1-\frac{\Lambda\left(z^{*}\right)}{k}, 1\right)$ such that

$$
\left(\frac{z^{I}}{z^{*}}\right)^{2}=\frac{\Lambda\left(z^{I}\right)}{\Lambda\left(z^{*}\right)}\left(1-\alpha^{*}\right)
$$

because $\left(\frac{z^{I}}{z^{*}}\right)^{2}<1<\frac{\Lambda\left(z^{I}\right)}{k}$. Hence, $E_{x}\left[q^{I}\right]=E_{x}\left[q^{*}\right]$ when $\alpha=\alpha^{* * *}$.

Case (c): $k<\Lambda\left(z^{*}\right)$. There exists $\alpha^{* *} \in(0,1)$ such that

$$
\left(\frac{z^{I}}{z^{*}}\right)^{2}=\frac{\Lambda\left(z^{I}\right)}{\Lambda\left(z^{*}\right)}\left(1-\alpha^{* *}\right)
$$

because $\left(\frac{z^{I}}{z^{*}}\right)^{2}<1<\frac{\Lambda\left(z^{I}\right)}{\Lambda\left(z^{*}\right)}$. Hence, $E_{x}\left[q^{I}\right]=E_{x}\left[q^{*}\right]$ when $\alpha=\alpha^{* *}$.

Furthermore, it is easy to verify that the investment level can also be coordinated in this situation. In general, the supply chain can achieve coordination in some certain level of overconfidence when $c>\frac{p}{2}$.

When $w<\frac{p}{2}$, we have $0<z^{*}<z^{I}$ and $\Lambda\left(z^{I}\right)<\Lambda\left(z^{*}\right)$. In this situation, there are three cases to consider. That is 
Case (a): $\Lambda\left(z^{*}\right) \leq k$. There is no $\alpha^{* *} \in\left(1-\frac{\Lambda\left(z^{*}\right)}{k}, 1\right)$ such that

$$
\left(\frac{z^{I}}{z^{*}}\right)^{2}=\frac{k}{\Lambda\left(z^{*}\right)}\left(1-\alpha^{* *}\right)
$$

because $\left(\frac{z^{I}}{z^{*}}\right)^{2}>1$. Hence, we have $E_{x}\left[q^{I}\right] \neq E_{x}\left[q^{*}\right]$ for any $\alpha$.

Case (b): $\Lambda\left(z^{I}\right) \leq k<\Lambda\left(z^{*}\right)$. There is no $\alpha^{* *} \in(0,1)$ such that

$$
\left(\frac{z^{I}}{z^{*}}\right)^{2}=\frac{k}{\Lambda\left(z^{*}\right)}\left(1-\alpha^{* *}\right)
$$

because $\left(\frac{z^{I}}{z^{*}}\right)^{2}>1>\frac{k}{\Lambda\left(z^{*}\right)}$. Hence, we have $E_{x}\left[q^{I}\right] \neq E_{x}\left[q^{*}\right]$ for any $\alpha$.

Case (c): $k<\Lambda\left(z^{I}\right)$. There is no $\alpha^{* *} \in(0,1)$ such that

$$
\left(\frac{z^{I}}{z^{*}}\right)^{2}=\frac{\Lambda\left(z^{I}\right)}{\Lambda\left(z^{*}\right)}\left(1-\alpha^{* *}\right)
$$

because $\left(\frac{z^{I}}{z^{*}}\right)^{2}>1>\frac{\Lambda\left(z^{I}\right)}{\Lambda\left(z^{*}\right)}$. Hence, we have $E_{x}\left[q^{I}\right] \neq E_{x}\left[q^{*}\right]$ for any $\alpha$.

In general, the supply chain cannot achieve coordination when $w<\frac{p}{2}$.

In contrast to the standard result of classic models, Proposition 4 indicates that when the total profit margin is low (i.e., $c>\frac{p}{2}$ ), the supply chain can be achieved under the wholesale price contract. Thus, overconfidence is a bias that can offset the effect of vertical decentralization such that a supply chain is equivalent to its centralized benchmark. In other words, wholesale price contracts can be an effective tool to coordinate the supply chain when the retailer is overconfident.

\section{Conclusions}

To the best of our knowledge, the previous literature studies information acquisition problems by assuming that decision makers are unboundedly rational. This work contributes to the emerging literature on behavioral operations management by considering a supply chain in which a supplier sells to a retailer, who is overconfident when he invests in acquiring information. Our analysis yields several interesting results.

First, our work shows that overconfidence does not necessarily cut the investment in information acquisition. In some situations, overconfidence gives the retailer the propensity to invest more in information acquisition, and causes the retailer to overinvest in information acquisition. Second, we show that over- 
confidence hurts the retailer's profits, and even eliminates the benefit of information. However, this bias can have a positive effect on the supplier's performance. Finally, we demonstrate that overconfidence with endogenous information acquisition can coordinate the supply chain. In presence of overconfidence, therefore, channel coordination can be achieved under the wholesale price contract.

\section{References}

[1] Oliveira, H., Denti, T., Mihm, M. and Ozbek, K. (2017) Rationally Inattentive Preferences and Hidden Information Costs. Theoretical Economics, 12, 621-654. https://doi.org/10.3982/TE2302

[2] Kartik, N., Lee, F.X. and Suen, W. (2017) Investment in Concealable Information by Biased Experts. The RAND Journal of Economics, 48, 24-43. https://doi.org/10.1111/1756-2171.12166

[3] Li, L., McKelvey, R.D. and Page, T. (1987) Optimal Research for Optimal Research for Cournot Oligopolists. Journal of Economic Theory, 42, 140-166. https://doi.org/10.1016/0022-0531(87)90107-4

[4] Vives, X. (1988) Aggregation of Information in Large Cournot Markets. Econometrica: Journal of the Econometric Society, 56, 851-876. https://doi.org/10.2307/1912702

[5] Hwang, H.S. (1993) Optimal Information Acquisition for Heterogenous Duopoly Firms. Journal of Economic Theory, 59, 385-402. https://doi.org/10.1006/jeth.1993.1024

[6] Fu, Q. and Zhu, K. (2010) Endogenous Information Acquisition in Supply Chain Management. European Journal of Operational Research, 201, 454-462. https://doi.org/10.1016/j.ejor.2009.03.019

[7] Shin, H. and Tunca, T.I. (2010) Do Firms Invest in Forecasting Efficiently? The Effect of Competition on Demand Forecast Investments and Supply Chain Coordination. Operations Research, 58, 1592-1610. https://doi.org/10.1287/opre.1100.0876

[8] Chen, F., Lai, G. and Xiao, W. (2015) Provision of Incentives for Information Acquisition: Forecast-Based Contracts vs. Menus of Linear Contracts. Management Science, 62, 1899-1914. https://doi.org/10.1287/mnsc.2015.2193

[9] Makris, M. and Siciliani, L. (2013) Optimal Incentive Schemes for Altruistic Providers. Journal of Public Economic Theory, 15, 675-699. https://doi.org/10.1111/jpet.12047

[10] Shin, D. (2017) Optimal Loyalty-Based Management. Journal of Economics \& Management Strategy, 26, 429-453. https://doi.org/10.1111/jems.12194

[11] Johnson, D.D. and Fowler, J.H. (2011) The Evolution of Overconfidence. Nature, 477, 317-320. https://doi.org/10.1038/nature10384

[12] Bensch, D., Paulhus, D.L., Stankov, L. and Ziegler, M. (2017) Teasing Apart Overclaiming, Overconfidence, and Socially Desirable Responding. Assessment, 1073191117700268. [Epub Ahead of Print]

[13] Cieślik, J., Kaciak, E. and van Stel, A. (2018) Country-Level Determinants and Consequences of Overconfidence in the Ambitious Entrepreneurship Segment. International Small Business Journal, 0266242617748201. [Epub Ahead of Print]

[14] Hribar, P. and Yang, H. (2016) CEO Overconfidence and Management Forecasting. Contemporary Accounting Research, 33, 204-227. 
https://doi.org/10.1111/1911-3846.12144

[15] Li, M., Petruzzi, N.C. and Zhang, J. (2016) Overconfident Competing Newsvendors. Management Science, 63, 2637-2646. https://doi.org/10.1287/mnsc.2016.2469

[16] Ren, Y. and Croson, R. (2013) Overconfidence in Newsvendor Orders: An Experimental Study. Management Science, 59, 2502-2517. https://doi.org/10.1287/mnsc.2013.1715

[17] Croson, R. and Donohue, K. (2006) Behavioral Causes of the Bullwhip Effect and the Observed Value of Inventory Information. Management Science, 52, 323-336. https://doi.org/10.1287/mnsc.1050.0436

[18] Barberis, N. and Thaler, R. (2003) A Survey of Behavioral Finance. Handbook of the Economics of Finance, 1, 1053-1128. https://doi.org/10.1016/S1574-0102(03)01027-6

[19] Ancarani, A., Di Mauro, C. and D’Urso, D. (2016) Measuring Overconfidence in Inventory Management Decisions. Journal of Purchasing and Supply Management, 22, 171-180. https://doi.org/10.1016/j.pursup.2016.05.001

[20] Tong, J. and Feiler, D. (2016) A Behavioral Model of Forecasting: Naïve Statistics on Mental Samples. Management Science, 63, 3609-3627. https://doi.org/10.1287/mnsc.2016.2537

[21] Ko, K.J. and Huang, Z.J. (2007) Arrogance Can Be a Virtue: Overconfidence, Information Acquisition, and Market Efficiency. Journal of Financial Economics, 84, 529-560. https://doi.org/10.1016/j.jfineco.2006.03.002

[22] Porteus, E.L. (2002) Foundations of Stochastic Inventory Theory. Stanford University Press, Redwood City. 